\title{
Tourism Route Plan Algorithm Based on Sight Spot Buffer Motive Iteration
}

\author{
Xiao Zhou ${ }^{1}$, Kuiliang Gao ${ }^{1, *}$, Yingcai Lun ${ }^{1}$, Jiang Zhu², Zhenning Yang ${ }^{2}$ and Qi Tong ${ }^{1}$ \\ ${ }^{1}$ Information Engineering University, No.62 Kexue Avenue, High-tech Zone, Zhengzhou, China \\ ${ }^{2}$ Army Academy of Border and Costal Defence, Xi'an, China \\ ${ }^{*}$ Corresponding author
}

\begin{abstract}
Currently, tourism plan cannot fully consider tourists' individual interests. And routes planned cannot meet tourists' best motive benefits. As to the problems, this paper brings forward tourism route plan algorithm basing on sight spot buffer motive iteration. Firstly, sight spot buffer model is set up and sight attracting factors and indexes are confirmed, on which basis, sight spot interval iteration model is set up. Through test example, optimal and suboptimal tourism routes which meet individual interests and best motive benefits are obtained. Example proves that this algorithm model can fully consider tourists' interests and output tourism routes to meet tourists' motive benefits, which is feasible and practical.
\end{abstract}

Keywords—sight spot buffer; tourism motive; motive iteration; route plan

\section{INTRODUCTION}

Tourists need to plan their tourism routes before visiting a city. Currently, urban tourism route plan has many limitations. Generally, tourists obtain tourism information and route information by purchasing books, visiting websites or travel agencies passively. It is difficult to extract valuable information which can satisfy tourists' motive benefits from mass information. Tourism routes are mainly based on the interest of the mainstream, which has a great blindness and can hardly meet the interests of individuals. As to the existing problems, this paper brings forward tourism route plan algorithm based on sight spot buffer motive iteration to meet the individualized needs of tourists. Based on the individualized needs of tourists, firstly, this algorithm sets up a sight spot buffer model. Then, taking advantage of sight spot interval motive factor, this algorithm calculates the output value of the motive benefits iteratively and views the route which has the highest motive benefits as the optimal tourism routes. The algorithm can reasonably output the tourism route which meets the individualized needs of tourists, which is feasible and practical.

\section{SIGHT SPOT BUFFER AND SigHT SPOT INTERVAL MOTIVE ITERATION MODEL}

Urban sight spots are concentrated holistically and independent individually, and located in different geographical locations. The attraction of urban sight spots is determined by many factors, such as, star level, comprehensive evaluation index, accommodation convenience, traffic convenience, consumption convenience and so on. Tourists' motivation to visit a spot results from multiple factors.
- Definition 1. Sight attracting factors $\delta$. The attraction index of a sight spot determined by the star level, comprehensive evaluation index, accommodation convenience, traffic convenience and consumption convenience.

Sight attracting factors are determined in a certain buffer. A buffer is a certain object's spatial neighborhood. The size of the neighborhood is determined by the radius or the conditions. Thus, as to a certain object $\mathrm{K}$, its buffer can be defined as follows:

$$
P=\{x \mid d(x, K) \leq r\}
$$

That is, the buffer of the object $K$ whose radius is $r$, is the set of all the points whose distance $d$ to $K$ is less than $r$. Generally, $d$ is the smallest Euclidean distance, but it also can be another defined distance.

Taking the sight spot as the center and $r$ as the radius, a sight spot buffer is set up. In the buffer, we can search for the number of hotel $t_{1}$, the number of the bus and subway station $t_{2}$, and the number of store and mall $t_{3}$. By combining star level $k_{1}$, and comprehensive evaluation index $k_{2}$, sight attracting factors $\delta$ and indexes $I_{\delta}$ are confirmed.

$$
\begin{gathered}
t_{i} \in[0, \infty] \in \mathrm{R}^{+}, k_{1} \in(0,5] \in \mathrm{Z}^{+}, k_{2} \in(0,10] \in \mathrm{R}^{+} . \\
I_{\delta}=\sum_{i=1}^{3} 0.01 t_{i}+\sum_{j=1}^{2} 0.01 k_{j}
\end{gathered}
$$

After choosing $n$ sight spots $N_{a}$ according to their own interests and hobbies, usually, tourists do one-way and nonrepeated browse in a certain order during a limited time. That is, one sight spot is only browsed once. Theoretically, there are $A_{n}^{n}$ tourism routes, however, not all tourism routes are the best. From the beginning spot to the ending spot, tourism routes are different, which influence the satisfaction of tourists. 
- Definition 2. Sight spot interval motive influence factor $Z_{u}$. Factors affecting the motivation of tourists during the tourism, $u \in(0, \max u] \in \mathrm{Z}^{+}$. It mainly includes the interval distance $S$, the number of the routes of the bus and the subway $q$, the cost of taxi $C$ and the number of traffic lights $l$.

Taking $\max u=4$ as an example, each sight spot interval motive influence factor index is shown in Table I.

TABLE I. SIGHT SPOT INTERVAL MOTIVE INFLUENCE FACTOR INDEX

\begin{tabular}{|c|c|c|c|c|}
\hline$z_{u}$ & $u=1$ & $u=2$ & $u=3$ & $u=4$ \\
\hline Val. & $s^{-1}$ & $0.1 q$ & $c^{-1}$ & $l^{-1}$ \\
\hline
\end{tabular}

- Definition 3. Sight spot interval motive iteration value $W_{b}$. The comprehensive index produced by the iteration of each motive factor between two sight spots, which has an influence on tourists' motivation, from the previous sight spot to the next sight spot, and $b \in(0, n-1] \in \mathrm{Z}^{+}$. Sight spot interval motive iteration model is shown in Formula 3 based on sight spot buffer model and sight attracting factors $\delta$.

$$
w_{b}=\sum_{u=1}^{\max u} w_{0} z_{u}+w_{0} I_{\delta}
$$

- Definition 4. Tourism route motive iteration value $W$. The total amount of iteration values which all the sight spots output from the beginning spot to the ending spot. Tourism route motive iteration model is shown in Formula 4 based on sight spot buffer model and sight attracting factors $\delta$.

$$
w=\sum_{b=1}^{n-1} w_{b}
$$

\section{AlgORITHM MODEL DESIGN}

Firstly, tourists choose $n$ sight spots according to their own interests and hobbies. Then, the system calculates the iteration value of the tourism motive intelligently. Taking Zhengzhou city as an example, the algorithm has been given as follows.

\section{A. Set up Sight Spot Buffer Model}

Taking the main sight spots in the city as the center, a sight spot buffer model with a certain radius has been set up. At the same time, sight attracting factors $\delta$ and indexes $I_{\delta}$ are obtained. Figure 1 shows several representative sight spot buffer and interval distance. The distance between spot $N_{a}$ and spot $N_{a^{\prime}}$ is $d_{a a^{\prime}}$. Table 2 shows sight attracting factors $\delta$ and indexes $I_{\delta}$.

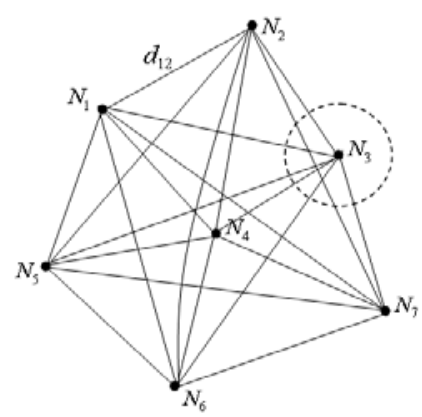

FIGURE I. SEVERAL REPRESENTATIVE SIGHT SPOT BUFFER AND INTERVAL DISTANCE

TABLE II. SIGHT ATTRACTING FACTORS $\delta$ AND INDEXES $I_{\delta}$

\begin{tabular}{|c|c|c|c|c|c|c|}
\hline$\delta$ & $t_{1}$ & $t_{2}$ & $t_{3}$ & $k_{1}$ & $k_{1}$ & $I_{\delta}$ \\
\hline$N_{1}$ Water Park & 8 & 2 & 3 & 2 & 2 & 0.170 \\
\hline$N_{2}$ Henan Museum & 7 & 3 & 4 & 5 & 4 & 0.230 \\
\hline $\begin{array}{c}N_{3} \text { Chinese Redbud } \\
\text { Mountain }\end{array}$ & 12 & 6 & 5 & 3 & 4 & 0.300 \\
\hline$N_{4}$ Memorial Hall & 19 & 6 & 8 & 4 & 5 & 0.420 \\
\hline $\begin{array}{c}N_{5} \text { Zhengzhou Science } \\
\text { and Technology Museum }\end{array}$ & 10 & 5 & 3 & 3 & 4 & 0.250 \\
\hline$N_{6}$ WanDa plaza, & 9 & 3 & 6 & 2 & 3 & 0.230 \\
\hline$N_{7}$ Zhongyuan Fortaleza & 6 & 2 & 2 & 4 & 4 & 0.180 \\
\hline
\end{tabular}

B. Calculate Sight Spot Interval Motive Iteration Value and Tourism Route Motive Iteration Values

As to the $n$ selected sight spots, there are $A_{n}^{n}$ tourism routes. As to the first tourism route, sight spot interval motive iteration value and tourism route motive iteration model $W_{1}$ are calculated. Similarly, the remaining $A_{n}^{n}-1$ tourism route motive iteration values are calculated. The bubble sort algorithm is as follows:

- Step.1 Construct the iteration value bubble vector $\vec{W}$ with $A_{n}^{n}$ elements numbers.

- Step.2 Calculate the first route and get $w_{1}$, and count $=1$

- Step.3 Calculate the second route and get $w_{2}$. If $w_{1}>w_{2}$, place $w_{1}$ in the first elements number of $\vec{W}$ and place $W_{2}$ in the second elements number of $\vec{W}$; if $w_{2}>w_{1}$, place $w_{2}$ in the first elements 
number of $\vec{W}$ and place $W_{1}$ in the second elements number of $\vec{W}$, and count $=2$.

- Step.4 Calculate the third route and get $w_{3}$. Go through and compare $w_{1}, w_{2}$ and $w_{3}$. Place the maximum value in the first elements number of $\vec{W}$. Place the second maximum value in the second elements number. Place the minimum value in the third elements number and count $=3$.

- Step.5 And so on, when count $=A_{n}^{n}$, the algorithm ends. There are $A_{n}^{n}$ results are obtained by the bubble sort. Thus, the iteration value bubble vector is obtained.

According to results, the route which is placed in the first element number of the iteration value bubble vector $\vec{W}$ is the optimal route. The optimal route fully considers the individualized needs of tourists, and can maximize their motive benefits. The route which is placed in the second element number of the iteration value bubble vector $\vec{W}$ is the suboptimal route.

\section{EXAMPLE ANALysis}

Take the representative sight spots in table 2 as an example, a tourist chooses to visit the Henan museum $N_{2}$, Zhengzhou science and technology museum $N_{5}$ and Wanda plaza $N_{6}$ in one day, according to his own interests. There are 6 visiting sequences. An iteration value bubble vector with six element numbers is constructed $\vec{W}=\left[w_{1}, w_{2}, w_{3}, w_{4}, w_{5}, w_{6}\right]$. Sight spot interval motive influence factor index is obtained according to the geographic information of sight spots interval. By combining sight attracting factors $\delta$ and indexes $I_{\delta}$, the system bubble output tourism route motive iteration values. The results are shown in Table 3.

TABLE III. SIGHT SPOT INTERVAL MOTIVE INFLUENCE FACTOR INDEX AND TOURISM ROUTE MOTIVE ITERATION VALUE

\begin{tabular}{|c|c|c|c|c|c|}
\hline$N$ & $Z_{1}$ & $Z_{2}$ & $Z_{3}$ & $Z_{4}$ & $w$ \\
\hline $2-5-6$ & $0.122 / 0.222$ & $0.500 / 0.500$ & $0.063 / 0.091$ & $0.067 / 0.111$ & 2.156 \\
\hline 2-6-5 & $0.102 / 0.222$ & $0.500 / 0.500$ & $0.053 / 0.091$ & $0.050 / 0.111$ & 2.109 \\
\hline 5-2-6 & $0.122 / 0.102$ & $0.500 / 0.500$ & $0.063 / 0.053$ & $0.067 / 0.050$ & 1.917 \\
\hline $5-6-2$ & $0.222 / 0.102$ & $0.500 / 0.500$ & $0.091 / 0.053$ & $0.111 / 0.050$ & 2.089 \\
\hline $6-2-5$ & $0.102 / 0.122$ & $0.500 / 0.500$ & $0.053 / 0.063$ & $0.050 / 0.067$ & 1.937 \\
\hline 6-5-2 & $0.222 / 0.122$ & $0.500 / 0.500$ & $0.091 / 0.063$ & $0.111 / 0.067$ & 2.156 \\
\hline
\end{tabular}

Through calculating iteratively and bubble sort, we can find out that, both visiting sequence 2-5-6 and 6-5-2 have the highest motive iteration value of tourism routes. It is easier for tourists to obtain the greatest motive benefits satisfaction according to the two tourism routes. Next is the visiting sequence 2-6-5, which is the suboptimal route. Tourists can choose the tourism route which is the most suitable for themselves according to the schedule and their own interests.

Figure 2 is sequence of the two optimal routes and one suboptimal route. Figure 3 is the guide maps of the two optimal routes and one suboptimal route.

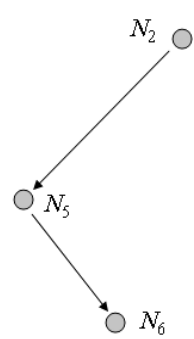

N2-5-6

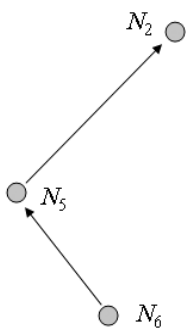

N6-5-2

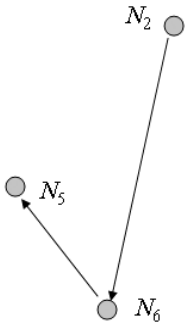

N2-6-5
FIGURE II. OPTIMAL AND SUBOPTIMAL ROUTE SEQUENCES
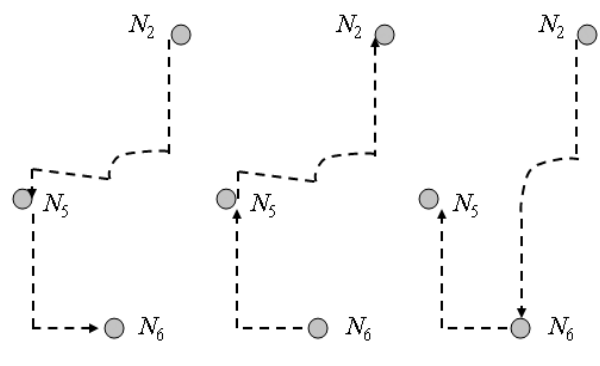

N2-5-6
N2-6-5

FIGURE III. OPTIMAL AND SUBOPTIMAL ROUTE GUIDE MAP

According to tour sequence and guide map, proper and feasible tour tips are put forward as follows.

- Tip.1 If tourists consider visiting venues in the morning while going shopping and relax in the afternoon, they can choose the tour sequence $N_{2} \rightarrow N_{5} \rightarrow N_{6}$ and get the best motive benefits.

- Tip.2 If tourists consider going shopping and relax in the morning while visiting venues in the afternoon, they can choose the tour sequence $N_{6} \rightarrow N_{5} \rightarrow N_{2}$ and get the best motive benefits.

- Tip.3 If tourists consider visiting the two venues in the morning and in the afternoon respectively while having lunch and relax at noon, they can choose the tour sequence $N_{2} \rightarrow N_{6} \rightarrow N_{5}$. Though the two venues are separated, yet tourists can have a good rest at noon.

\section{CONCLUSION}

This paper brings forward tourism route plan algorithm based on sight spot buffer motive iteration, based on the existing problems of tourism route plan. Through setting up the sight spot buffer and sight spot interval motive iteration model, the system calculates iteratively and bubble output the optimal tourism route which has the best iteration value. Through the 
analysis of the examples, this algorithm model can fully consider tourists' interests and output tourism routes to meet tourists' motive benefits, which is feasible and practical.

\section{REFERENCES}

[1] Gimblett R, Daniel T, Cherry S, Meitner M J ( 2001 ). The simulation and visualization of complex human-environment interactions. Landscape and Urban Planning, 54( 1-4), 63-78.

[2] Rossello-Nadal J(2001). Forecasting turning points in international visitor arrivals in the Balearic Islands. Tourism Economics'7'365-380.

[3] Graburn N( 1977). Tourism: The Sacred Journey. Philadelphia: University of Pennsylvania Press, 19-36.

[4] Itami R, R aulings R, Maclarcn G, et al. ( 2003) . R BSim 2: Simulation the complex interactions between human movement and the outdoor recreation environment. Journal of Nature Conservation, 11(4), 278-286.

[5] Manuel V, Robertico R C( 2000). Evaluation of demand US tourists to Aruba. Annals of Tourism Research'27( 4), 946-963.

[6] Zhang J, Gu F, Lu F P. Analysis and Optimization of City Tourism Spatial Behavior Path:A Case Study of Nanjing. Geography and GeoInformation Science, 2011, 27(1): 85-89.

[7] Pan J H, Li J F. Analysis on Spatial Structure of A-Grade Scenic Spots in China Based on Quantitative Geography Model. Economic Geography, 2013, 33(9): 154-160. doi: 10.3969/j.issn.10008462.2013.09.024

[8] Zhao X, Guan Z H, Wang H. An Analysis on Choice Behavior of Tourist Destination Based on Tourist Motivation. Journal of Transportation Systems Engineering and Information Technology, 2014, 14(5): 175-180. doi: 10.3969/j.issn.1009-6744.2014.05.026

[9] Zheng T X,Wu R. Decision Support System to Personalized Route Guidance Service for Tourists in Theme Parks Based on Computer Reasoning Technology: Design, Simulation and Comparison. Tourism Science, 2016, 30(1):60-74.

[10] Xu F, Du J P. Study on travel route planning based on improved ant colony algorithm. Computer Engineering and Applications, 2009, 45(23) 193-195. 